\title{
Predictive value of inflammatory indexes on the chemotherapeutic response in patients with unresectable lung cancer: A retrospective study
}

\author{
HAIFENG SUN ${ }^{1,3}$, PINGPING HU ${ }^{2}$, JIAJUN DU ${ }^{3}$ and XINYING WANG ${ }^{1}$ \\ ${ }^{1}$ Research Institute of General Surgery, Jinling Hospital, Medical School of Nanjing University, Nanjing, Jiangsu 210002; \\ ${ }^{2}$ Department of Radiation Oncology, Qianfoshan Hospital Affiliated to Shandong University; \\ ${ }^{3}$ Department of Thoracic Surgery, Shandong Provincial Hospital Affiliated to Shandong University,
} Jinan, Shandong 250000, P.R. China

Received December 4, 2016; Accepted November 16, 2017

DOI: $10.3892 / \mathrm{ol} .2018 .7781$

\begin{abstract}
Chemotherapy is widely administered to patients with advanced lung cancer; however, data regarding chemotherapeutic sensitivity are limited. The present study aimed to investigate the predictive value of inflammatory indexes for chemotherapeutic efficacy in advanced lung cancer. Patients with stage III and IV unresectable lung cancer that were treated with first-line chemotherapy between January 2007 and December 2011 were retrospectively identified, and chemotherapeutic response was evaluated following 2 or 3 chemotherapy cycles. Prior to chemotherapy, hematologic data and clinicopathological parameters were collected using electronic medical records. The associations between the main inflammatory indexes [which included the pretreatment neutrophil count (PNC), neutrophil-lymphocyte ratio (NLR), platelet-lymphocyte ratio (PLR)] and the chemotherapeutic efficacy, as well as the prognostic value of the indexes, were analyzed. According to the receiver operating characteristic curve, PLR failed to reach diagnostic accuracy for overall chemotherapeutic response. PNC and NLR were each classified into two groups according to the cut-off values $\left(4.635 \times 10^{9} / 1\right.$ for PNC and $2.443 \times 10^{9} / 1$ for NLR). The overall response rate was significantly higher in the low PNC [odds ratio, 3.261; 95\%
\end{abstract}

Correspondence to: Professor Xinying Wang, Research Institute of General Surgery, Jinling Hospital, Medical School of Nanjing University, 305 East Zhongshan Road, Nanjing, Jiangsu 210002, P.R. China

E-mail: wxinying@263.net

Professor Jiajun Du, Department of Thoracic Surgery, Shandong Provincial Hospital Affiliated to Shandong University, 324 Jingwu Road, Jinan, Shandong 250000, P.R. China

E-mail: haifeng6629@163.com

Key words: pretreatment neutrophil count, neutrophil-lymphocyte ratio, platelet-lymphocyte ratio, chemotherapeutic response, chemoresistance, lung cancer confidence interval (CI), 2.102-5.060; P<0.001, vs. high PNC] and low NLR groups (odds ratio, 1.596; 95\% CI, 1.037-2.454; $\mathrm{P}=0.033$, vs. high NLR). Univariate analyses showed that the high PNC (HR, 1.487) and high NLR groups (HR, 1.288) were associated with poor progression-free survival (PFS); however, NLR was considered statistically insignificant in multivariate analysis. In summary, high PNC and NLR values are associated with chemoresistance and an unfavorable prognosis, with the present study demonstrating that PNC has increased sensitivity when compared with other inflammatory indexes in predicting chemotherapeutic efficacy. Therefore, PNC has the potential to be used as a reliable and suitable predictor to stratify a high risk of chemoresistance in patients with stage III and IV unresectable lung cancer.

\section{Introduction}

Lung cancer remains the most common and lethal malignant tumor type worldwide, despite extensive research and numerous clinical trials $(1,2)$. The first therapeutic choice for patients with early-stage lung cancer is surgical excision. However, in the majority of cases, tumors have developed to the unresectable stage by the time of initial diagnosis, and surgical resection is no longer a viable option (3). Thus, systemic chemotherapy has become the principal treatment for lung cancer. However, it possesses limitations with regard to its efficacy, a prime example being that patients rarely survive for an extended time period following treatment. In this regard, identifying increasingly sensitive markers for predicting chemotherapeutic efficacy, and therapeutic targets to promote the development of individualized treatment has become a popular area of research.

The mechanisms underlying resistance to anticancer agents may be categorized into two types, comprising tumor cell intrinsic factors and non-tumor cell intrinsic factors. The former includes increased drug efflux by ATP-binding cassette superfamily proteins, dose-associated toxicities, increased DNA repair mechanisms, apoptosis deficiency and drug alteration (4-7). However, these insights into drug resistance have not thus far led to major improvements in the survival rates of patients with lung cancer, who usually exhibit immune suppression 
and cancer-associated inflammation (8). For instance, inflammatory leukocytes, together with proteolytic enzymes and dysregulated vessels form the tumor microenvironment. High levels of-tumor-associated macrophages (TAM), one of the most important types of inflammatory leukocytes (9), exhibit a crucial role in the chemotherapy-resistance of patients with lung cancer (10). Furthermore, cancer-associated fibroblasts have been demonstrated to contribute to the doxorubicin-resistance of breast cancer cells (11). Gene polymorphisms in DNA repair pathways and multi-drug resistance gene 1 (MDR1) could also contribute to the response of non-small cell lung cancer (NSCLC) to chemotherapy (12).

In the 19th century, Rudolf Virchow (13) identified the association between inflammation and cancer. Thereafter, studies confirmed an extensive association between inflammation and cancer (14-16). Cancer may induce an inflammatory state, resulting in multiple inflammatory cells being recruited to the cancer microenvironment, and contributing to the hallmarks of cancer $(16,17)$. Notably, indexes of inflammatory cells [including the pretreatment neutrophil count (PNC), macrophage, neutrophil-lymphocyte ratio (NLR) and platelet-lymphocyte ratio (PLR)] in the bloodstream can reflect the scope and extent of inflammation $(18,19)$. Previous studies have reported that elevations in the PNC, NLR or PLR indicate a poor prognosis in various human cancer types, most notably in colorectal cancer (19), hepatocellular carcinoma (20), and in gastric (21), esophageal (22), breast $(23,24)$, ovarian (25), cervical (26) and lung cancer $(27,28)$. Increasingly, studies have suggested that inflammation serves an important role in the regulation of chemoresistance (8,29-32). This is consistent, with the notion that certain inflammatory indexes correlate with chemotherapeutic responses; for example, NLR correlates with chemotherapeutic responses in breast cancer (33). Van Glabbeke et al (34) demonstrated that an elevated baseline neutrophil count correlated with initial, as well as late, resistance to imatinib treatment in gastrointestinal stromal tumors (GIST). However, studies regarding the association between chemotherapeutic sensitivity and inflammatory indexes are scarce, particularly in lung cancer. Despite this, we hypothesized that common inflammatory indexes could serve as important predictors for chemotherapeutic response. The available literature leaves certain questions unanswered, such as which indexes may be used for predicting the response to chemotherapy in lung cancer, and which index is superior.

The primary aim of this study was to investigate and compare the predictive value of commonly used inflammatory indexes on chemotherapeutic efficacy in advanced lung cancer.

\section{Materials and methods}

Ethical approval. This protocol of this retrospective study was reviewed and approved by the Ethics Committee of Shandong Provincial Hospital Affiliated to Shandong University, and written informed consent was obtained from all participants for their clinical records to be used in this study.

Patient and data collection. All participating patients with stage III and IV unresectable lung cancer (NSCLC and SCLC) received chemotherapy as the initial treatment between January 2007 and December 2011 at the Department of Oncology, Shandong Provincial Hospital Affiliated to Shandong University (Jinan, China) and Jinling Hospital (Nanjing, China) were retrospectively identified from the hospital's original electronic databases. The primary inclusion criteria were as follows: i) Definitive diagnosis of primary lung cancer; ii) explicit pathological pattern before treatment; and iii) chemotherapy as a first-line therapy without prior treatment. The exclusion criteria were as follows: i) Patients with a history of other types of cancer; ii) patients whose data were incomplete; iii) patients for whom chemotherapeutic efficacy was not evaluated; and iv) patients who received concurrent radiochemotherapy prior to response evaluation.

Progression-free survival (PFS) was defined as the time period from the start of treatment to the date that tumor progression was detected. The patients' clinicopathological data were collected using electronic medical records. This, clinicopathological information included sex, age, inflammatory manifestation, smoking status, obstructive pneumonia, central tumor location, clinical stage, chemotherapeutic response, overall response and histopathological pattern. PNC, NLR and PLR were calculated from the medical records at the time of the first explicit diagnosis prior to chemotherapy.

Response assessment. Chemotherapeutic response was assessed after 2 or 3 chemotherapeutic cycles using the revised Response Evaluation Criteria in Solid Tumors (version 1.1) (34). The criteria classified the responses into four categories: Complete response (CR), partial response (PR), stable disease (SD) and progressive disease (PD). CR was defined as the complete disappearance of all measurable lesions sustained for $\geq 4$ weeks. PR was defined as a minimum $30 \%$ reduction in measurable lesions sustained for $\geq 4$ weeks. SD was defined as a $<30 \%$ decrease or $<20 \%$ increase in the size of measurable lesions. PD was assigned to patients when measurable lesions increased by $>20 \%$, or when new lesions were identified (35). Overall response included $\mathrm{CR}$ and $\mathrm{PR}$. The overall response rate was the ratio of overall response patients to the total patients.

Statistical analysis. All statistical analyses were performed using SPS version 20.0 (IBM Corp., Armonk, NY, USA). Analysis of inflammatory indexes was performed according to the overall response by receiver operating characteristic (ROC) curves, which were used to detect the value of each index for predicting the response to chemotherapy. Associations between inflammatory indexes and clinicopathological parameters were investigated using the $\chi^{2}$ test. A nonparametric test of numerical variables was used for testing differences in the distribution of inflammatory indexes among different groups. PFS was estimated using the Kaplan-Meier method and the log-rank test was used for comparison of outcomes. The Cox proportional hazards regression model was used to confirm independent predictors of PFS, and multivariate Cox analyses were performed with a step-forward logistic regression approach. $\mathrm{P}<0.05$ was considered to indicate a statistically significant difference.

\section{Results}

Patient characteristics. All the main clinicopathological characteristics of samples are detailed in Table I. A total 


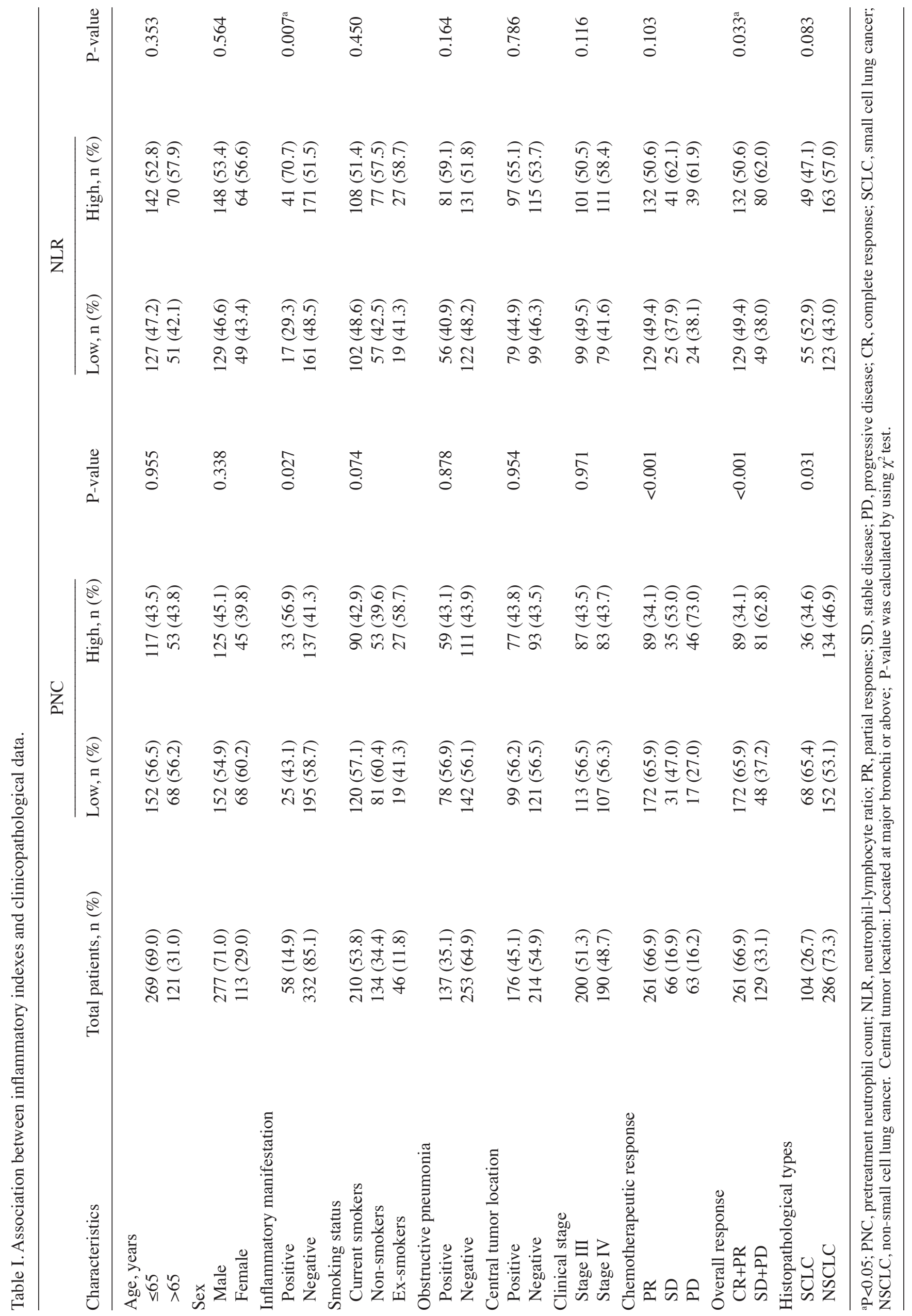


A

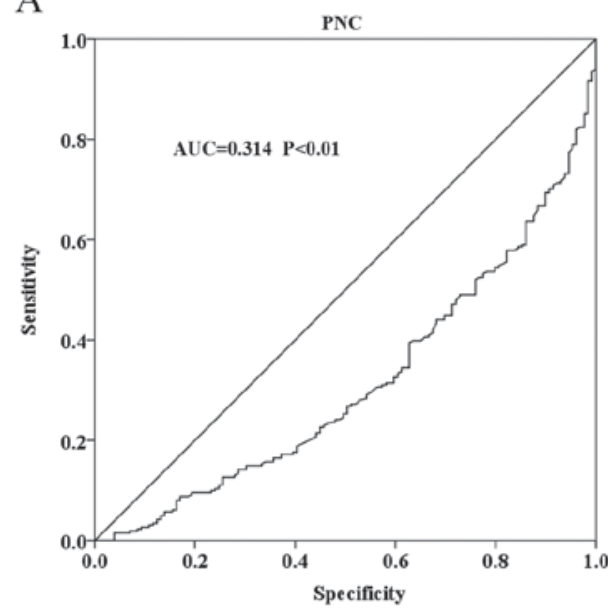

B

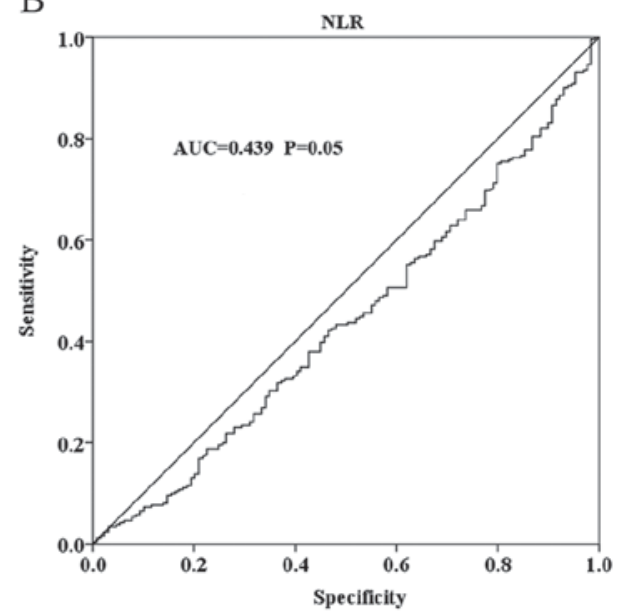

Figure 1. Diagnostic value of inflammatory indexes for overall response according to ROC curves. (A) The optimal cut-off point for PNC was $4.635 x 109 / 1$, AUC was 0.314 with $\mathrm{P}<0.01$; (B) The optimal cut-off point of NLR was 2.443 , the AUC was 0.439 where $\mathrm{P}=0.05$. ROC, receiver operating characteristic; AUC, area under curve; PNC, pretreatment neutrophil count.

of 390 patients, based on the original electronic files, were retrospectively enrolled in the study. All samples were from patients with stage III or IV unresectable lung cancer who received platinum-based chemotherapy as a first-line treatment. There were 277 (71.0\%) males and 113 (29.0\%) females, with $121(31.0 \%)$ patients $>65$ years of age and the other $269 \leq 65$ (69.0\%; range, $18-84$ years). There were 58 (14.9\%) patients with inflammatory manifestation (fever, yellow sputum, purulent sputum, etc.) while this was not present in the other $332(85.1 \%)$. In terms of clinical tumor stage, 200 $(51.3 \%)$ patients had stage III and 190 (48.7\%) had stage IV disease. Regarding chemotherapeutic response, no patients achieved CR, 261 achieved PR, 66 had SD and 63 had PD. In total 261 patients $(66.9 \%)$ achieved an overall response, and the other 129 patients $(33.1 \%)$ had no marked chemotherapeutic response. PNC and NLR were divided into two levels by the critical values, and PLR did not indicate significance according to the ROC curve.

The association between inflammatory indexes and overall response rate. The cut-off values based on the ROC curve were calculated as $4.635 \times 10^{9} / 1$ for PNC and 2.443 for NLR. However, the PLR failed to reach diagnostic accuracy for the overall response (Fig. 1). According to the cut-off values, PNC and NLR were each divided into low and high groups. The ROC curve indicated that PNC [area under curve (AUC), 0.314; $\mathrm{P}<0.001$ )] and NLR (AUC, 0.439; $\mathrm{P}=0.05$ ) were significant predictors of overall response. The overall response rate was $78.2 \%(172 / 220)$ in the low PNC group, and only $52.4 \%$ $(89 / 170)$ in the high PNC group (odds ratio, 3.261; 95\% CI, $2.102-5.060 ; \mathrm{P}<0.001)$. The overall response rate was $72.5 \%$ $(129 / 178)$ in the low NLR group, and was reduced to $62.3 \%$ (132/212) in high group (odds ratio, 1.596; 95\% CI, 1.037-2.454; $\mathrm{P}=0.033)$.

Associations between inflammatory indexes and clinicopathological characteristics. The associations between the clinicopathological parameters and inflammatory indexes are presented in Table I. High levels of PNC were found to be significantly associated with inflammatory manifestation $(\mathrm{P}=0.027)$, non overall response ( $\mathrm{SD}+\mathrm{PD} ; \mathrm{P}<0.001)$, chemotherapeutic response $(\mathrm{P}<0.001)$ and histopathological types $(\mathrm{P}=0.031)$. Conversely, there was no significant association between PNC level and other clinical parameters, such as age $(\mathrm{P}=0.955)$, sex $(\mathrm{P}=0.338)$, smoking status $(\mathrm{P}=0.074)$, obstructive pneumonia $(\mathrm{P}=0.878)$, central tumor location $(\mathrm{P}=0.954)$ or clinical tumor stage $(\mathrm{P}=0.971)$. NLR was significantly associated with inflammatory manifestation $(\mathrm{P}=0.007)$ and overall response $(\mathrm{P}=0.033)$; however, there was no significant association with other parameters.

Distribution of inflammatory indexes according to chemotherapeutic response and overall response. To analyze differences in the distribution of PNC and NLR between patients who exhibited a chemotherapeutic response and overall response, a Mann-Whitney U test was used. Comparison of the PNC and NLR values among the three chemotherapeutic response groups revealed that the differences were statistically significant $(\mathrm{PR}<\mathrm{SD}, \mathrm{P}=0.008 ; \mathrm{SD}<\mathrm{PD}, \mathrm{P}=0.001 ; \mathrm{PR}<\mathrm{PD}, \mathrm{P}<0.001)$ (Fig. 2A). Consistently, patients with low PNC were more likely to exhibit an overall response to chemotherapy $(\mathrm{P}<0.001$; Fig. 2B). However, the distribution of NLR demonstrated no significant difference (Fig. 2C and D). Further analyses, revealed the predictive efficiency of $\mathrm{PNC}$ for overall response existed in the NSCLC $(\mathrm{P}<0.001)$ and SCLC $(\mathrm{P}<0.001)$ subgroups (Fig. 3).

Survival analyses. The last follow-up was performed in December 2014, and 24 (6.2\%) patients were lost to follow up. In the present study, PFS was the endpoint for the entire cohort. Kaplan-Meier survival curves based on PNC and NLR levels are shown in Fig. 4. Statistically significant differences in survival were identified between the different levels of PNC $(\mathrm{P}<0.001)$ and NLR $(\mathrm{P}=0.016)$; high levels of PNC and NLR were significantly associated with recurrence risk and poor prognosis. The Cox proportional hazards regression model was used for confirming the independent predictors of PFS (Table II). According to univariate Cox regression 

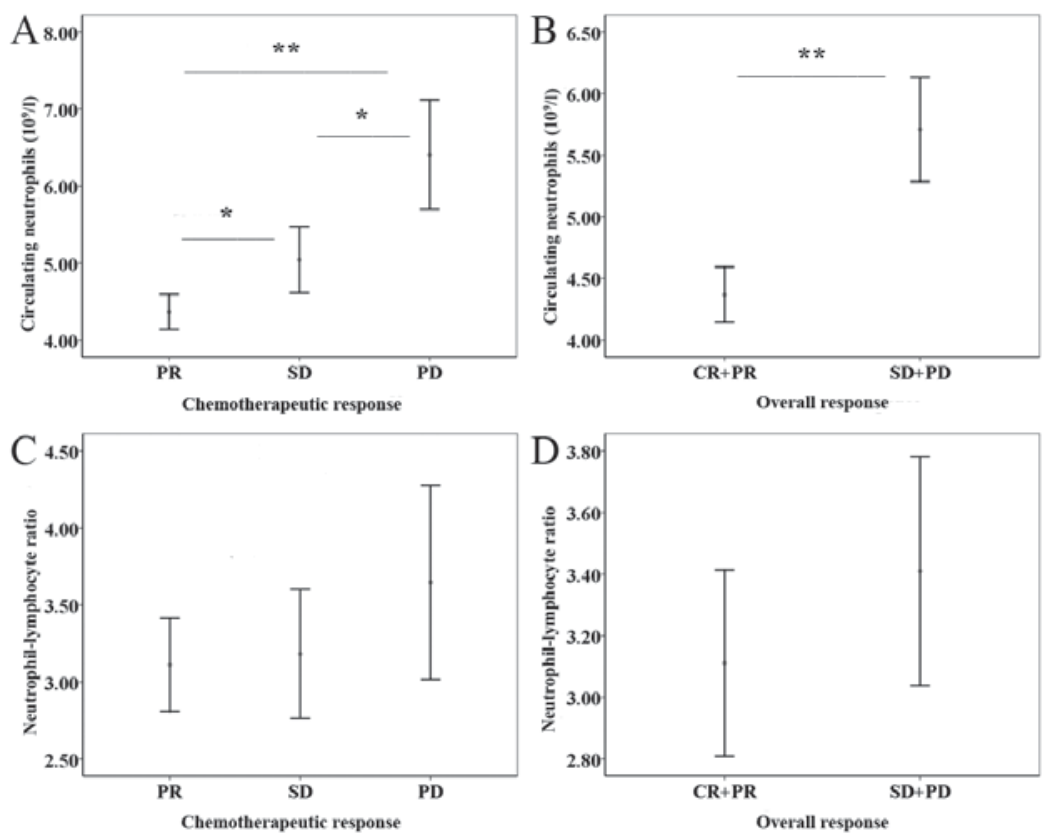

Figure 2. Distribution of PNC and NLR. Distribution of PNC according to (A) chemotherapeutic response and (B) overall response. Distribution of NLR according to (C) chemotherapeutic response and (D) overall response. PR, partial response; $\mathrm{SD}$, stable disease; $\mathrm{CR}$, complete response. ${ }^{*} \mathrm{P}<0.01$ and ${ }^{* *} \mathrm{P}<0.001$.
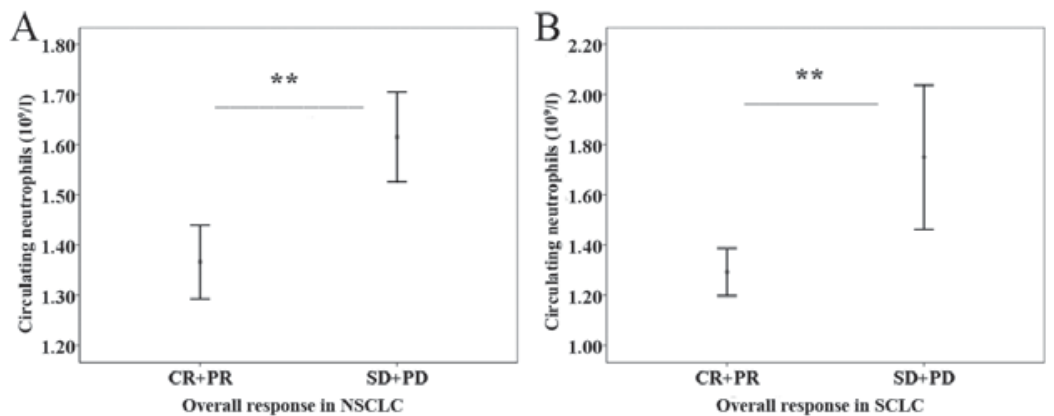

Figure 3. Distribution of pretreatment neutrophil count in (A) NSCLC and (B) SCLC. NSCLC, non-small cell lung cancer; SCLC, small cell lung cancer. ${ }^{* *} \mathrm{P}<0.001$.

analysis, PFS was correlated with NLR levels (HR, 1.288; 95\% CI, 1.041-1.594; $\mathrm{P}=0.020$ ), PNC levels (HR, 1.487, 95\% CI, 1.200-1.841; $\mathrm{P}<0.001)$, overall response (HR, 2.349; 95\% CI, 1.878-2.939; $\mathrm{P}<0.001)$ and clinical tumor stage (HR, 1.537, 95\% CI=1.241-1.904; $\mathrm{P}<0.001)$. No significant associations were identified between survival and sex, age or smoking status. Multivariate Cox analysis confirmed that PNC levels, overall response and clinical stage were independent predictors of PFS ( $\mathrm{P}=0.011, \mathrm{P}<0.001$ and $\mathrm{P}=0.001$, respectively).

\section{Discussion}

Millions of individuals worldwide receive a lung cancer diagnosis each year, and the majority of the cases are detected, whereas the disease has developed into unresectable tumor stage $(1,2,36)$. Chemotherapy serves crucial role in the treatment of unresectable lung cancer (3); however, chemoresistance has become a vital factor that negatively influences curative effects. Therefore, there is an urgent requirement to identify powerful biomarkers associated with the chemotherapeutic response that can contribute to selecting optimal therapies for individuals. Therefore, the present study attempted to establish the relevance of certain inflammatory indexes to chemotherapeutic efficacy in 390 patients with stage III or IV unresectable lung cancer. To the best of our knowledge, this is the first study to identify and compare chemotherapeutic efficacy among PNC, NLR and PLR in advanced lung cancer.

Links between systemic inflammation and cancer have garnered academic interest and have been the focus of numerous studies (14-16). Emerging evidence suggests that systemic inflammation and the tumor-associated inflammatory microenvironment serve an important additional role in modulating chemotherapeutic responsiveness and chemoresistance; however, the underlying mechanisms remain largely unclear $(8,29)$. Hematological markers of systemic inflammation, including C-reactive protein, PNC, NLR, PLR, albumin-neutrophil prognostic grade etc., are well established as useful in the prediction of outcomes in a number of cancer types (19-23,25-28,37-40). Nevertheless, there is a paucity of studies regarding the associations between inflammatory indexes and chemotherapeutic response. Furthermore, the conclusions of these studies have been inconsistent. For 
Table II. Cox proportional hazard's analyses.

Univariate

Characteristics

Sex

Female

Male

Age (years)

$>65$

$\leq 65$

1 (ref.)

$1.024(0.817-1.283)$

Smoking status

Current smokers

Non-smokers

1 (ref.)

1 (ref.)

0.253

1.145 (0.908-1.444)

Ex-smokers

NLR levels

Low

High

Circulating neutrophil levels

Low

High

Overall response

$\mathrm{CR}+\mathrm{PR}$

$\mathrm{SD}+\mathrm{PD}$

Clinical stage

Stage III

Stage IV
$0.941(0.748-1.183)$

$1.056(0.750-1.487)$

1 (ref.)

1.288 (1.041-1.594)

1 (ref.)

1.487 (1.200-1.841)

1 (ref.)

2.349 (1.878-2.939)

1 (ref.)

1.537 (1.241-1.904)
0.837

0.783

$0.020^{\mathrm{a}}$

$<0.001^{\mathrm{a}}$

$<0.001^{\mathrm{a}}$

$<0.001^{\text {a }}$
Multivariate

P-value

HR (95\% CI)

P-value

HR $(95 \% \mathrm{CI})$

.

${ }^{\mathrm{a}}<0.05$; -, not included in the final step of multivariate analysis; $\mathrm{CI}$, confidence interval; HR, hazard ratio; NLR, neutrophil-lymphocyte ratio $\mathrm{CR}$, complete response; PR, partial response; SD, stable disease; PD, progressive disease. P-values were calculated using $\chi^{2}$ test.

A

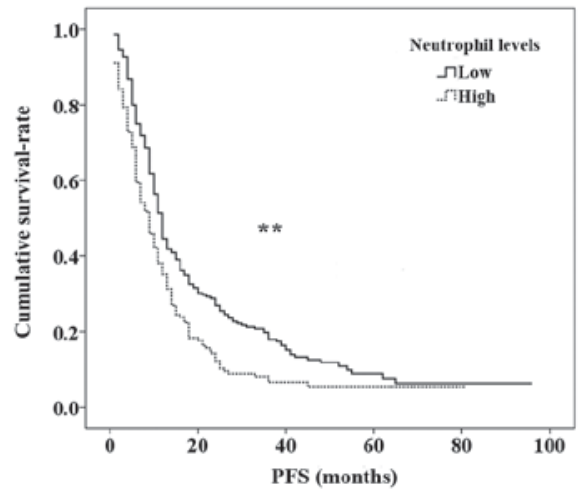

B

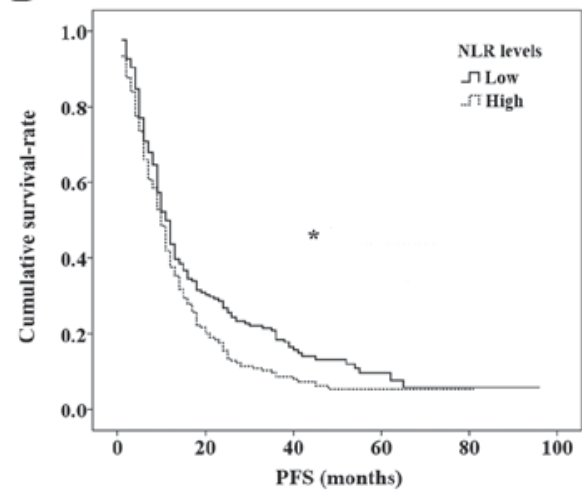

Figure 4. Kaplan-Meier curves show PFS according to (A) the pretreatment neutrophil count and (B) the NLR. PFS, progression-free survival. "P $<0.05$ and ${ }^{* *} \mathrm{P}<0.001$.

example, Van Glabbeke et al (34) identified that elevated PNC was correlated with chemoresistance in GIST. An additional study demonstrated that a normal neutrophil ratio was independently associated with the response to chemotherapy in SCLC (41). Previous research also demonstrated that NLR was correlated with chemotherapeutic response $(33,42)$. Conversely, Eryilmaz et al (43) demonstrated no significant association between CR and NLR levels. In the present study, it was confirmed that PNC and NLR were significant predictors of chemotherapeutic response. Notably, PNC presented an increased sensitivity value, compared with NLR and PLR, for predicting chemoresistance. Therefore, PNC has the potential to function as a novel and powerful factor for predicting chemotherapeutic efficacy in lung cancer. 
Hematological inflammatory cells are indispensable components of the tumor microenvironment, which is analogized to 'soil supporting the growth of plants', to support tumor progression and chemoresistance $(8,15)$. Firstly, tumor-infiltrating neutrophils (TINs), the most important 'fertilizers' in the 'soil' $(8,15)$, lose their conventional antitumor characteristics and acquire a pro-tumor phenotype in the presence of transforming growth factor- $\beta$ (TGF- $\beta$ ) (44). In our previous study, TIN count was revealed to serve as a prognostic factor and to promote epithelial-mesenchymal transition (EMT) in esophageal cancer (45), which may be a key process involved in regulating chemoresistance in malignant tumors (46-48). However, TIN is not a conventional indicator for detection due to difficulty in its measurement. Furthermore, PNC is largely recruited via chemoattractant mediators, including chemokines, lipids, complement anaphylotoxins and $\mathrm{N}$-formylated peptides into tumor microenvironment, and then is converted into TIN (49). PNC is correlated with TIN in quantitative terms (27); therefore, PNC can indirectly reflect and influence the chemotherapeutic response. Elevated PNC can stimulate upregulation of cytokines and chemokines (13), and this confers cancer cells with acquired resistance to chemotherapeutic drugs $(8,50,51)$. These possible mechanisms are consistent with the present results: Elevated PNC is significantly associated with a poor chemotherapeutic response and poor prognosis in patients with advanced lung cancer. NLR is the ratio of the PNC to the pretreatment lymphocyte count, and therefore the association between high NLR and poor chemotherapeutic response, as revealed in the present study, may indicate that chemoresistance is associated with neutrophilia. However, lymphocytes destroy not only invading pathogens but also malignancies, via the induction of cytotoxic death. A consequential decrease in the lymphocyte count may lead to a weaker immune reaction against cancer cells $(15,52)$. Additionally, neutrophilia suppresses lymphocyte activity by releasing reactive oxygen species (ROS), nitric oxide (NO) and arginase (23), therefore hindering the antitumor immune response (21). As established, systemic inflammation associated immune suppression is the predominant non-tumor-cell-intrinsic mechanism of chemoresistance (8). The present study demonstrated that individuals in the high NLR group have an increased risk of experiencing a poor response to chemotherapy and a poor PFS time. Finally, PLR (the ratio of platelets to lymphocytes,) was not significantly associated with chemotherapeutic response in the present study, despite two previous studies obtaining the opposite results $(28,53)$.

In the study, the associations between common inflammatory indexes and the chemotherapeutic response and clinicopathological parameters, in addition the outcome of patients with stage III or IV unresectable lung cancer, were investigated. PLR failed to indicate a statistically significant result. According to previous studies, high PNC and NLR were associated with poor PFS and a lower rate of response to chemotherapy. The novel and notable finding from this data that may have practical implications is that high PNC exhibits a higher overall response and HR than NLR. Furthermore, significant distributional differences in PNC were identified in the different chemotherapy response groups and overall response groups, whereas the NLR distribution did not significantly differ. This indicates that PNC may be more powerful and sensitive in predicting chemotherapeutic response. This may be due to the superior sensitivity of neutrophils for indicating inflammatory states, and their direct participation in cancer-associated inflammatory microenvironments. An additional reason may be that different ages, tumor stages and histopathological phenotypes correspond with different immune responses, and therefore hematological data varies.

Additionally, the PNC distribution according to overall response was also examined in different histopathological subtypes. The results revealed that the significant differences in distribution were universal in NSCLC and SCLC, despite the differences between the two in terms of biological properties and therapeutic measures.

The major limitations of the present study are as follows: First, numerous individuals were excluded due to incomplete data or the unsuccessful completion of follow-up, which may have led to selective bias; second, patients with different types of lung cancer exhibited different immune responses and different inflammatory states, and a stratified analysis of each subtype was not conducted; third, this study failed to investigate more inflammatory indexes, such as the Glasgow prognostic score (GPS); furthermore, conclusions were solely drawn from the objective clinical data, as it was beyond the scope of our study to elucidate the mechanism of association between inflammation and chemoresistance. The combined aforementioned limitations suggest that the results require validation in additional independent cohorts of patients with specific lung cancer types, ideally through large-scale prospective clinical studies.

In summary, the present study indicated that PNC and NLR were clinically important predictors of chemotherapeutic response in patients with stage III and IV unresectable lung cancer who received chemotherapy as first-line treatment. Additionally, PNC represents a more robust indicator for chemoresistance compared with other inflammatory indexes. This study has the potential to provide a highly reproducible, easily obtainable, inexpensive, reliable and practical index for predicting chemotherapeutic efficacy, and to facilitate the administration of therapy in patients with a high PNC in order to reach an improved chemotherapeutic response, thereby enhancing the long-term outcomes for patients with unresectable lung cancer. However, the potential underlying mechanisms and the performance of PNC in clinical practice should be validated in further prospective studies.

\section{References}

1. Torre LA, Bray F, Siegel RL, Ferlay J, Lortet-Tieulent J and Jemal A: Global cancer statistics, 2012. Ca Cancer J Clin 65: 87-108, 2015

2. Siegel R, Naishadham D and Jemal A: Cancer statistics, 2013. CA Cancer J Clin 63: 11-30, 2013.

3. Wu L, Chang W, Zhao J, Yu Y, Tan X, Su T, Zhao L, Huang S, Liu $\mathrm{S}$ and Cao G: Development of autoantibody signatures as novel diagnostic biomarkers of non-small cell lung cancer. Clin Cancer Res 16: 3760-3768, 2010.

4. Schiller JH, Harrington D, Belani CP, Langer C, Sandler A, Krook J, Zhu J and Johnson DH; Eastern Cooperative Oncology Group: Comparison of four chemotherapy regimens for advanced non-small-cell lung cancer. N Engl J Med 346: 92-98, 2002.

5. Chang A: Chemotherapy, chemoresistance and the changing treatment landscape for NSCLC. Lung Cancer 71: 3-10, 2011. 
6. Raymond E, Chaney SG, Taamma A and Cvitkovic E: Oxaliplatin: A review of preclinical and clinical studies. Ann Oncol 9: 1053-1071, 1998.

7. Borst P, Jonkers J and Rottenberg S: What makes tumors multidrug resistant? Cell Cycle 6: 2782-2787, 2007.

8. de Visser KE and Jonkers J: Towards understanding the role of cancer-associated inflammation in chemoresistance. Current Pharm Des 15: 1844-1853, 2009.

9. Solinas G, Germano G, Mantovani A and Allavena P: Tumor-associated macrophages (TAM) as major players of the cancer-related inflammation. J Leukoc Biol 86: 1065-1073, 2009.

10. De Palma M and Lewis CE: Macrophage regulation of tumor responses to anticancer therapies. Cancer Cell 23: 277-286, 2013.

11. Amornsupuk K, Insawang T, Thuwajit P, O-Charoenrat $\mathrm{P}$, Eccles SA and Thuwajit C: Cancer-associated fibroblasts induce high mobility group box 1 and contribute to resistance to doxorubicin in breast cancer cells. BMC cancer 14: 955, 2014.

12. Du Y, Su T, Zhao L, Tan X, Chang W, Zhang H and Cao G: Associations of polymorphisms in DNA repair genes and MDR1 gene with chemotherapy response and survival of non-small cell lung cancer. PLoS One 9: e99843, 2014.

13. Balkwill $\mathrm{F}$ and Mantovani A: Inflammation and cancer: Back to Virchow? Lancet 357: 539-545, 2001.

14. Grivennikov SI, Greten FR and Karin M: Immunity, inflammation and cancer. Cell 140: 883-899, 2010.

15. Coussens LM and Werb Z: Inflammation and cancer. Nature 420 : $860-867,2002$

16. Hanahan D and Weinberg RA: Hallmarks of cancer: The next generation. Cell 144: 646-674, 2011.

17. Hanahan D and Coussens LM: Accessories to the crime: Functions of cells recruited to the tumor microenvironment. Cancer cell 21: 309-322, 2012.

18. Carus A, Gurney H, Gebski V, Harnett P, Hui R, Kefford R, Wilcken N, Ladekarl M, von der Maase H and Donskov F: Impact of baseline and nadir neutrophil index in non-small cell lung cancer and ovarian cancer patients: Assessment of chemotherapy for resolution of unfavourable neutrophilia. J Transl Med 11: 189, 2013.

19. Zou ZY, Liu HL, Ning N, Li SY, Du XH and Li R: Clinical significance of pre-operative neutrophil lymphocyte ratio and platelet lymphocyte ratio as prognostic factors for patients with colorectal cancer. Oncol Lett 11: 2241-2248, 2016.

20. Lai Q, Castro Santa E, Rico Juri JM, Pinheiro RS and Lerut J: Neutrophil and platelet-to-lymphocyte ratio as new predictors of dropout and recurrence after liver transplantation for hepatocellular cancer. Transpl Int 27: 32-41, 2014.

21. Lee S, Oh SY, Kim SH, Lee JH, Kim MC, Kim KH and Kim HJ Prognostic significance of neutrophil lymphocyte ratio and platelet lymphocyte ratio in advanced gastric cancer patients treated with FOLFOX chemotherapy. BMC Cancer 13: 350, 2013.

22. Feng JF, Huang Y and Chen QX: Preoperative platelet lymphocyte ratio (PLR) is superior to neutrophil lymphocyte ratio (NLR) as a predictive factor in patients with esophageal squamous cell carcinoma. World J Surg Oncol 12: 58, 2014

23. Azab B, Shah N, Radbel J, Tan P, Bhatt V, Vonfrolio S, Habeshy A, Picon A and Bloom S: Pretreatment neutrophil/lymphocyte ratio is superior to platelet/lymphocyte ratio as a predictor of long-term mortality in breast cancer patients. Med Oncol 30: 432, 2013.

24. Krenn-Pilko S, Langsenlehner U, Thurner EM, Stojakovic T, Pichler M, Gerger A, Kapp KS and Langsenlehner T: The elevated preoperative platelet-to-lymphocyte ratio predicts poor prognosis in breast cancer patients. Br J Cancer 110: 2524-2530, 2014.

25. Asher V, Lee J, Innamaa A and Bali A: Preoperative platelet lymphocyte ratio as an independent prognostic marker in ovarian cancer. Clin Transl Oncol 13: 499-503, 2011.

26. Carus A, Ladekarl M, Hager H, Nedergaard BS and Donskov F: Tumour-associated CD66b + neutrophil count is an independent prognostic factor for recurrence in localised cervical cancer. Br J Cancer 108: 2116-2122, 2013

27. Carus A, Ladekarl M, Hager H, Pilegaard H, Nielsen PS and Donskov F: Tumor-associated neutrophils and macrophages in non-small cell lung cancer: No immediate impact on patient outcome. Lung Cancer 81: 130-137, 2013.

28. Liu H, Wu Y, Wang Z, Yao Y, Chen F, Zhang H, Wang Y and Song Y: Pretreatment platelet-to-lymphocyte ratio (PLR) as a predictor of response to first-line platinum-based chemotherapy and prognosis for patients with non-small cell lung cancer. J Thorac Dis 5: 783-789, 2013
29. DeNardo DG, Brennan DJ, Rexhepaj E, Ruffell B, Shiao SL, Madden SF, Gallagher WM, Wadhwani N, Keil SD and Junaid SA: Leukocyte complexity predicts breast cancer survival and functionally regulates response to chemotherapy. Cancer Discov 1: 54-67, 2011

30. Muccioli M and Benencia F: Toll-like receptors in ovarian cancer as targets for immunotherapies. Front Immunol 5: 341, 2014.

31. Jinushi $\mathrm{M}$ and Komohara Y: Tumor-associated macrophages as an emerging target against tumors: Creating a new path from bench to bedside. Biochim Biophys Acta 1855: 123-130, 2015.

32. Delitto D, Black BS, Sorenson HL, Knowlton AE, Thomas RM, Sarosi GA, Moldawer LL, Behrns KE, Liu C, George TJ, et al: The inflammatory milieu within the pancreatic cancer microenvironment correlates with clinicopathologic parameters, chemoresistance and survival. BMC Cancer 15: 783, 2015.

33. Chen Y, Chen K, Xiao X, Nie Y, Qu S, Gong C, Su F and Song E: Pretreatment neutrophil-to-lymphocyte ratio is correlated with response to neoadjuvant chemotherapy as an independent prognostic indicator in breast cancer patients: A retrospective study. BMC Cancer 16: 320, 2016.

34. Van Glabbeke M, Verweij J, Casali PG,Le Cesne A,Hohenberger P, Ray-Coquard I, Schlemmer M, van Oosterom AT, Goldstein D, Sciot R, et al: Initial and late resistance to imatinib in advanced gastrointestinal stromal tumors are predicted by different prognostic factors: A european organisation for research and treatment of cancer-italian sarcoma group-australasian gastrointestinal trials group study. J Clin Oncol 23: 5795-5804, 2005.

35. Eisenhauer EA, Therasse P, Bogaerts J, Schwartz LH, Sargent D, Ford R, Dancey J, Arbuck S, Gwyther S, Mooney M, et al: New response evaluation criteria in solid tumours: Revised RECIST guideline (version 1.1). Eur J Cancer 45: 228-247, 2009.

36. Brower V: Biomarker studies abound for early detection of lung cancer. J Natl Cancer Inst 101: 11-13, 2009.

37. Sun H, Hu P, Shen H, Dong W, Zhang T, Liu Q and Du J: Albumin and neutrophil combined prognostic grade as a new prognostic factor in non-small cell lung cancer: Results from a large consecutive cohort. PLoS One 10: e0144663, 2015.

38. Kishida Y, Kawahara M, Teramukai S, Kubota K, Komuta K, Minato K, Mio T, Fujita Y, Yonei T, Nakano K, et al: Chemotherapy-induced neutropenia as a prognostic factor in advanced non-small-cell lung cancer: Results from japan multinational trial organization LC00-03. Br J Cancer 101: 1537-1542, 2009.

39. Schmidt H, Bastholt L, Geertsen P, Christensen IJ, Larsen S, Gehl J and von der Maase H: Elevated neutrophil and monocyte counts in peripheral blood are associated with poor survival in patients with metastatic melanoma: A prognostic model. Br J Cancer 93: 273-278, 2005.

40. Negrier S, Mejean A, Oudard S and Escudier B: (Metastatic kidney cancer: New therapeutic approaches). Prog Urol 12: 703-708, 2002

41. Paesmans M, Sculier JP, Lecomte J, Thiriaux J, Libert P, Sergysels R, Bureau G, Dabouis G, Van Cutsem O, Mommen P, et al: Prognostic factors for patients with small cell lung carcinoma: Analysis of a series of 763 patients included in 4 consecutive prospective trials with a minimum follow-up of 5 years. Cancer 89: 523-533, 2000.

42. Sato H, Tsubosa $Y$ and Kawano T: Correlation between the pretherapeutic neutrophil to lymphocyte ratio and the pathologic response to neoadjuvant chemotherapy in patients with advanced esophageal cancer. World J Surg 36: 617-622, 2012.

43. Eryilmaz MK, Mutlu H, Salim DK, Musri FY, Tural D and Coskun HS: The neutrophil to lymphocyte ratio has a high negative predictive value for pathologic complete response in locally advanced breast cancer patients receiving neoadjuvant chemotherapy. Asian Pac J Cancer Prev 15: 7737-7740, 2014.

44. Fridlender ZG, Sun J, Kim S, Kapoor V, Cheng G, Ling L, Worthen GS and Albelda SM: Polarization of tumor-associated neutrophil phenotype by TGF-beta: 'N1' versus 'N2' TAN. Cancer Cell 16: 183-194, 2009.

45. Hu P, Wang G, Shen M, Zhang P, Zhang J, Du J and Liu Q: Intratumoral polymorphonuclear granulocyte is associated with poor prognosis in squamous esophageal cancer by promoting epithelial-mesenchymal transition. Future Oncol 11: 771-783, 2015.

46. Hoshino H, Miyoshi N, Nagai K, Tomimaru Y, Nagano H, Sekimoto M,Doki Y,Mori M and Ishii H: Epithelial-mesenchymal transition with expression of SNAI1-induced chemoresistance in colorectal cancer. Biochem Biophys Res Commun 390: 1061-1065, 2009. 
47. Kurrey NK, Jalgaonkar SP, Joglekar AV, Ghanate AD, Chaskar PD, Doiphode RY and Bapat SA: Snail and slug mediate radioresistance and chemoresistance by antagonizing p53-mediated apoptosis and acquiring a stem-like phenotype in ovarian cancer cells. Stem Cells 27: 2059-2068, 2009.

48. Canadas I, Rojo F, Taus Á, Arpí O, Arumí-Uría M, Pijuan L, Menéndez S, Zazo S, Dómine M and Salido M: Targeting epithelial-to-mesenchymal transition with Met inhibitors reverts chemoresistance in small cell lung cancer. Clin Cancer Res 20 938-950, 2014.

49. Dumitru CA, Lang S and Brandau S: Modulation of neutrophil granulocytes in the tumor microenvironment: Mechanisms and consequences for tumor progression. Semin Cancer Biol 23: 141-148, 2013.
50. Kelly MG, Alvero AB, Chen R, Silasi DA, Abrahams VM, Chan S, Visintin I, Rutherford T and Mor G: TLR-4 signaling promotes tumor growth and paclitaxel chemoresistance in ovarian cancer. Cancer Res 66: 3859-3868, 2006.

51. Nakanishi $\mathrm{C}$ and Toi M: Nuclear factor-kappaB inhibitors as sensitizers to anticancer drugs. Nat Rev Cancer 5: 297-309, 2005.

52. Mantovani A, Allavena P, Sica A and Balkwill F: Cancer-related inflammation. Nature 454: 436-444, 2008.

53. Miao Y, Yan Q, Li S, Li B and Feng Y: Neutrophil to lymphocyte ratio and platelet to lymphocyte ratio are predictive of chemotherapeutic response and prognosis in epithelial ovarian cancer patients treated with platinum-based chemotherapy. Cancer Biomark 17: 33-40, 2016. 